\title{
Proyectos participativos y trasversales como base de aprendizaje de una visión crítica y socialmente responsable de la comunicación
}

\author{
Rosa María TorRes VALDÉs \\ Universidad de Alicante \\ rosatorres@ua.es \\ Patricia NúÑEZ GómEZ \\ Universidad Complutense de Madrid \\ pnunezgo@ccinf.ucm.es \\ Liisa Irene HANNINEN \\ Universidad Complutense de Madrid \\ liisaire@ucm.es
}

\section{Resumen:}

El proyecto utiliza la metodología Investigación Acción para poner en marcha una campaña de comunicación real y analizar simultáneamente la motivación y percepciones de los alumnos participantes. La razón de ser de la iniciativa es explorar formulas para implicar a los estudiantes en los procesos de creación de conocimiento y enseñarles una visión socialmente responsable y crítica de la comunicación, además de ofrecerles una oportunidad de mejorar su CV mediante una experiencia profesional en colaboración con profesionales de la agencia y del cliente, expertos en salud y nutrición y profesores.

Palabras clave: Publicidad; visión crítica; proyecto multidisciplinar; generación de conocimiento; comunicación responsable; motivación.

\section{Participative and transversal projects as a learning base for acquiring a critical and socially responsible view of communication}

\begin{abstract}
:
The project applies the methodology of Action Research in order to carry out a real communication campaign and simultaneously, analyze the motivations and perceptions of the partaking students. The reason why is to explore new formulas for involving students in the process of generating knowledge and teaching them a socially responsible and critical point of view of communication, as well as to offer them an opportunity to complete their $\mathrm{CV}$ with a work experience in collaboration with specialized staff from the agency and client, health and nutrition experts as well as university lecturers.
\end{abstract}

Key Words: Advertising; critical point of view; multidisciplinary project; generating knowledge; responsible communications; motivation.

\section{Referencia normalizada:}

Torres Valdés, R. M., Nuñez Gómez, P. y Hanninen, L. I. (2014): Proyectos participativos y trasversales como base de aprendizaje de una visión crítica y socialmente responsable de la comunicación. Historia y Comunicación Social. Vol. 19. Núm. Especial Enero. Págs. 443-456. 
Sumario: 1. Introducción al estado de la cuestión. 2. Metodología. 3. Desarrollo del proyecto. 4. Conclusiones. 5. Referencias bibliográficas.

\section{Introducción al estado de la cuestión}

El hecho de buscar nuevas fórmulas docentes, que orienten y "profesionalicen" a los estudiantes, no está reñido con la orientación sobre la importancia y el rigor científico.

Los procesos educativos deben tener en cuenta también la creación de comunidades participativas: "es un reto necesario a asumir por todos los profesionales de la educación y la formación, dado que a través de ellas se puede, por una parte, transferir y generar nuevo conocimiento y por otra, lograr mejores prácticas en el campo de la investigación y la docencia” (Bozu \& Imbernón, 2009).

Las profesoras firmantes, que pertenecen a diferentes grupos de investigación de la Universidad de Alicante y de la Universidad Complutense, sustentan este trabajo de investigación como experiencia piloto

El trabajo forma parte de una investigación en curso entre el Grupo de Cuarta Cultura y Comunicación Científica de Proximidad de la Universidad de Alicante y el grupo Branding Kids de la Universidad Complutense de Madrid. Realizan desde hace varios años, una actividad docente, en la que se aborda la formación competencial en el ámbito informativo comunicativo, desde una perspectiva, transversal, multidisciplinar, desde el punto de vista ético y socialmente responsable. El reto fundamental está en la gestión comunicativa comprometida en un tema tan sensible como es la salud, que en el caso que se presenta tiene que ver con la publicidad y los hábitos alimenticios saludables de las personas en general y de los menores en particular.

Fomentando la visión crítica y multidisciplinar de los alumnos a través de un caso real e impartiendo conocimientos básicos de contenido de manera transversal construyen nuevos escenarios de conocimiento y experiencia y amplían su campo de visión en relación con el grado en que se están preparando, de modo que descubran nuevas oportunidades y nuevos campos de desarrollo de carrera profesional.

\subsection{Base del aprendisaje socialmente responsable}

Debemos orientar para preparar a los alumnos al panorama de incertidumbre (González \& Tiscar, 2011), y, además nuestros estudios de comunicación deben incluir valores de comunicación responsable y una visión multidisciplinar y crítica mediante el Diálogo de Saberes (Robles, 2005) y el paradigma de la Cuarta Cultura (Lehrer, 2010).

Autores como Olmedo Estrada (2011), señalan la importancia de la alfabetización científica en relación con una ciudadanía del S.XXI, informada y participativa. Olmedo expone en su análisis cinco conceptos fundamentales: "comunicación, 
cognición, responsabilidad social, contextualización, y participación ciudadana en la implantación de políticas públicas relacionadas con incorporaciones tecno-científicas en el devenir cotidiano.

En este estudio, se recogen los resultados del desarrollo de planes de comunicación estratégicos vinculados con la empres, con el mundo real y la sensibilización realizada con los alumnos con el objetivo de construir y educar ciudadanos responsables con los temas de la sociedad, en este caso, la salud y los más jóvenes.

Se trata de encaminar estrategias de comunicación y procesos de aprendizaje hacia una sociedad del futuro, más sostenible y basada en valores corresponsables. Aunque la planificación estratégica es parte del proyecto, la flexibilidad está presente en todas sus etapas, permitiendo aprender haciendo, con una visión de comunicación flexible y reaccionando a tiempo real a los cambios del entorno, Más allá de la interacción con el entorno social, la pro-actividad es una de las claves de éxito a la hora de diseñar campañas socialmente responsables.

Por otra parte, y de manera integral, además de forma $\mathrm{r}$ a los alumnos, la universidad debe ser socialmente responsable, cumpliendo así su misión social. Todo el proyecto recoge las tres estrategias socialmente responsables para lograr la mejora de la universidad que señala Vallaeys en su artículo sobre la responsabilidad social de la Universidad (2009: 209):

1) La participación integrada de los grupos de interés internos y extenso en el quehacer de la Universidad.

2) La articulación de planes de estudios, la investigación, la extensión y los métodos de enseñanza con la solución de los problemas de la sociedad;

3) El auto diagnóstico regular de la institución con herramientas apropiadas de medición para la rendición de cuentas hacia los grupos de interés.

Los métodos docentes interactivos son los que hemos estado trabajando durante años en nuestros equipos de investigación donde la empresa, la sociedad y la universidad están integradas de manera conjunta y permiten poner en práctica a nuestros estudiantes los conocimientos adquiridos, al aplicarlo a la situación profesional real pero con la supervisión y apoyo de los docentes. En este sentido, la forma de trabajar con los estudiantes huye de los modelos verticales y rígidos de aprendizaje mientras que se crean contextos de trabajo donde los profesores se convierten en mentores de los estudiantes.

En este caso, siendo alumnos de comunicación audiovisual y publicidad se realizó una campaña integral, basada por un lado, en los conceptos de responsabilidad social de las entidades (Mullerat, 2007: 50-54) y por otro, en el marketing social y la comunicación solidaria (Balas, 2011: 96-98; Kotler, Hessekiel y Lee, 2012: 171-172; Barranco Saiz, 2005: 29; Benet y Nos Aldás, 2003: 178), aparte de diversas obras de referencia en el campo de las relaciones públicas y organización de eventos (entre 
otros las publicaciones de Castillo, 2010; Juholin, 2006; Wilcox, Cameron y Xifra, 2006 y Xifra, 2007).

\subsection{Historia del equipo participante}

En la universidad Complutense, Facultad de Ciencias de la Información, el equipo tiene una amplia experiencia de 15 años con proyectos de innovación social y con técnicas de enseñanza interactivas aplicadas a las entidades del tercer sector. Los alumnos trabajan en planes estratégicos de entidades no lucrativas, con diferentes grados de responsabilidad dependiendo de los cursos donde están. En 2004, en el seno del proyecto, docentes y estudiantes pusieron en marcha el gabinete de comunicación para el Banco de Alimentos de Madrid, que el año siguiente sería el encargado de organizar el $10^{\circ}$ aniversario de la entidad y en 2014, ayudará con el 20 aniversario. Después, el equipo consiguió el reconocimiento del Rectorado de la Universidad Complutense de Madrid y desde entonces, el equipo ha trabajado con los fondos de innovación docente elaborando planes de investigación y comunicación para un centenar de entidades no lucrativas.

Paralelamente se organizan eventos en el campus con este sector donde colaboran los alumnos de manera práctica aplicando lo aprendido en las clases.

En Alicante, en 1994 se crea El Gabinete de Iniciativas Para el Empleo y Unidad de Responsabilidad Social. Desde 1993 ya en el marco de la asignatura Teoría y técnicas de las relaciones públicas se comenzó una línea de trabajo que fomentaba la orientación investigadora y aprendizaje activo-participativo por parte de los alumnos, dando todo ello lugar a actividades y prácticas en proyectos de Responsabilidad Social y comunicación responsable en ámbitos de diversidad cultural, diversidad funcional, género y envejecimiento activo y saludable. Entre otros resultados, cabe destacar el surgimiento de iniciativas emprendedoras que hoy día son empresas en activo.

De este conjunto de experiencias, surge el know how para aplicarlo en el ámbito de la empresa privada del sector hortofrutícola y alimentario, con productos dirigidos a niños.

La investigación con niños y medios de comunicación, forma parte muy importante de nuestro equipo, a través del grupo Branding kids, su relación con las marcas y la manera de relacionarse con los mercados como un consumidor crítico y responsable generó el inicio de este último proyecto.

\section{Metodología}

Si los alumnos forman parte de un proceso de investigación acción en el doble papel de observadores y observados, se estimulará su curiosidad investigadora multidisciplinar y se incrementará su capacidad de visión crítica sobre sistemas y procesos 
de comunicación. A través de este estudio, se demuestra, una vez más, que se puede estimular y motivar nuevas formas de hacer una comunicación socialmente responsable, de manera multidisciplinar y crítica.

Tomando como línea de contenidos de enseñanza, el ámbito de la salud y la comunicación, enfocados a la alimentación saludable, se les propone un problema comunicativo a resolver, transversalmente, les estamos enseñando de manera integral responsabilidad social y contenidos comunicativos, abordando valores éticos .

Se organizó una jornada técnica sobre comunicación para la salud con el tema "Información y sensibilización sobre prevención de la obesidad infantil y de riesgos para la salud de las personas, por malos hábitos alimenticios". En este sentido, la Investigación Acción o Action Research por su naturaleza comunicacional y de "investigación-intervención-observación participativa" nos ha permitido abordar la experiencia desde el trabajo en equipo profesores-alumnos, teórico-práctico.

Recurrimos a la Investigación Acción "La adecuación de la investigación-acción como forma de entender la educación y no sólo de indagar en ella ha sido recogida en la literatura pedagógica" (Romera-Iruela, 2011)

Finalmente se desarrolló un análisis cualitativo a partir de dinámicas de grupo presenciales y on line, de cuestionario post evento y de la vivencia durante todo el proceso planificador y organizativo.

En este caso puntual trabajamos con 12 alumnos, 4 alumnos de la asignatura de cuarto curso Teoría y Técnicas de las Relaciones Públicas del grupo Cuarta Cultura y comunicación científica de proximidad de la Universidad de Alicante, 4 alumnos de la Asignatura de Teoría de la Publicidad y Relaciones Públicas del Grupo Branding Kids de la Universidad complutense y 4 personas de la empresa cliente: director general, coordinador de comunicación, jefe de calidad y jefa de relaciones comerciales. Hablamos, por tanto de un muestreo intencional no probabilístico, para esta experiencia que en sí misma constituye una prueba piloto, de la investigación acción en base a un caso: el de la organización de jornadas sobre comunicación y salud como estrategia de Relaciones Públicas para el lanzamiento de producto alimenticio bajo la marca Power Pupis.

La aproximación, por lo tanto es cualitativa y el estudio es exploratorio como inicio de una investigación posterior para garantizar resultados y rigor metodológico. La combinación de técnicas cuanti y cualitativas definen nuestras investigaciones. La utilización de metodología cualitativa está justificada como medición de indicadores para desarrollar los ítems cuantitativos necesarios.

En este caso también, lo estudiamos de manera individual como un estudio de caso. La definición de Neiman y Quaranta (2006:220), en Kazez (2009) sobre el término caso nos indica lo siguiente: "El caso es definido como un sistema delimitado en el tiempo y espacio de actores, relaciones e instituciones sociales". Kazez (2009) explica que "el estudio de caso consiste en el abordaje de lo particular priorizando el 
caso único, en donde el estudio del mismo es definido por el interés que este inspira, mientras que el diseño metodológico del estudio es secundario".

Es por eso que tomamos la experiencia como una sola unidad aunque posteriormente nos sirva para desarrollar las demás investigaciones, la jornada técnica sobre comunicación para la salud, es nuestra unidad de análisis y nuestro laboratorio para plantear experiencias con mayor número de participantes. En este sentido coincidimos con Díaz de Salas y otros (2011) en que:

El Estudio de Caso parte del supuesto de que es posible conocer un fenómeno estudiado partiendo de la explicación intensiva de la unidad de análisis, donde el potencial heurístico está centrado en la relación entre el problema de investigación y la unidad de análisis, lo que facilita la descripción, explicación y compresión del sujeto/objeto de estudio. El investigador en el estudio de caso debe, en un inicio, reconocer la unidad/sujeto/objeto de estudio, que puede ser una persona, una organización, un programa de estudio, un acontecimiento particular o una unidad de análisis documental. Y entre las muchas ventajas de un estudio de caso, señalan que (...) si bien los estudios de casos no prueban hipótesis, sugieren direcciones para estudios subsecuentes.

\section{Sobre el proyecto y su desarrollo}

\subsection{Planteamientos generales diseño}

Para concretar la experiencia en este caso, describiremos brevemente de qué empresa se trata y que problema plantea al proyecto de investigación. La empresa pertenece al sector hortofrutícola: su nombre es GreenGrocer, que pretende lanzar al mercado un nuevo producto alimenticio a base de hortalizas frescas cuya marca es Power Pupis. La necesidad del cliente es llegar a las personas objeto de la comunicación y consumidores potenciales mediante estrategias comunicativas y relacionales. El problema: ¿Cómo hacerlo de modo responsable teniendo en cuenta que la alimentación está relacionada con la salud?

El trabajo se organizó como una empresa de comunicación real, organizada por departamentos y con el proceso de trabajo también real, es decir, desde el principio, entrega de información, contacto con el cliente, intervención de diferentes departamentos y corrección y supervisión de las profesoras.Los alumnos aprendieron a desarrollar las habilidades necesarias que se exigen en el mundo profesional, a la vez que se sensibilizan sobre temas sociales.

Este es el modelo generado por este grupo con experiencia en trabajos de este tipo, tales como campañas de integración, prevención de drogas y otros temas sociales.

En las diversas sesiones se trabajaron los siguientes temas: en la primera se preguntó a los alumnos sobre conceptos básicos relacionados con los temas de publicidad y comunicación para la salud. Y se les pidió una opinión sobre los mensajes 
publicitarios relacionados con alimentos. En la segunda sesión se expuso el caso y se les invitó a exponer sus ideas. En la tercera sesión, presencialmente se aborda el debate de la cuarta cultura, el conocimiento multidisciplinar, y la díada ciencia y arte, en el quehacer de publicidad y Relaciones Públicas, y cómo aplicar todo ello al caso Power Pupis.

Casi siempre nos ocurre y, en este caso es igual que los alumnos tienden a sugerir soluciones creativas sin estrategia ni investigación, así ellos ven la necesidad de investigar y formarse en esas áreas para dar la eficacia pedida. Los alumnos de ambas universidades trabajan on line las ideas gráficas y demás piezas Se desarrollan las diferentes sesiones para ayudarles a aprender cómo se debe trabajar en estos casos $\mathrm{y}$, con esa información ya son capaces de diseñar todo el trabajo real. Los alumnos participantes reconocen que sienten la necesidad de investigar tanto sobre normativa, como sobre información científica relacionada con la alimentación saludable y prevención de obesidad infantil, para seleccionar los medios de información, exponer la noticia, y seleccionar invitados prescriptores clave y consumidores a la jornada técnica sobre comunicación para la salud, con el tema específico "Información y sensibilización sobre prevención de la obesidad infantil y de riesgos para la salud de las personas, por malos hábitos alimenticios".

Este caso tuvo además la colaboración del programa "El estirón "en Antena3, permitiendo hacer acciones más globales de integración mediática y pudiendo planificar diferentes medios a la vez.

Todo está reflejado en la Jornada que se realizó en Madrid como punto final del proceso de aprendizaje colaborativo

\section{PROGRAMA PARA JORNADA DE PRESENTACIÓN EN MADRID JORNADA TÉCNICA SOBRE COMUNICACIÓN PARA LA SALUD.}

Tema específico: Información y sensibilización sobre prevención de la obesidad infantil y de riesgos para la salud de las personas, por malos hábitos alimenticios.

Organizan: Universidad Complutense de Madrid y Universidad de Alicante.

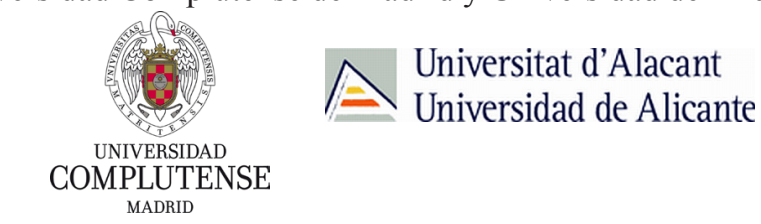

Patrocina: Greengrocer y Power Pupis
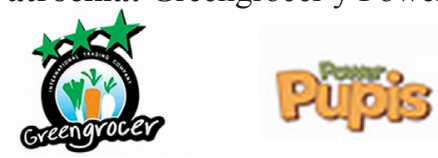


\section{Objetivos de la jornada:}

- Reunir a los profesionales de la salud en materia de nutrición y a profesionales de la comunicación, para la construcción colectiva de un glosario de términos y un prontuario de expresiones divulgativas en materia de prevención de la obesidad infantil y riesgos para la salud de las personas de todas las edades, a causa los malos hábitos alimenticios.

- Presentar un nuevo producto alimenticio producto de I+D de la empresa GreenGrocer al servicio de la alimentación saludable para todas las edades, exponer el nuevo enfoque de la estrategia de comunicación realizada basada en la filosofía de Responsabilidad Social GreenGrocer.

- Contribuir a la formación práctica de estudiantes de ciencias de la comunicación dando la oportunidad de participar en la organización del evento y de exponer su experiencia y lecciones aprendidas sobre comunicación relacionada con la salud.

\section{PROGRAMA:}

17:00 a 17:25: Acto inaugural.

\section{PRIMERA SESIÓN.}

17:30 a 18:00 Mesa de especialistas de la salud

Conocimientos fundamentales para comunicar sobre salud nutricional en general y prevención de la obesidad infantil en particular

\section{PONENTES:}

- Dra. Clotilde Vázquez. Jefe Sección Endocrinología y Nutrición Hospital Ramón y Cajal. Coordinadora de grupo de Obesidad de SENDIMAD (Sociedad de Endocrinología, Nutrición y Diabetes, de la Comunidad de Madrid). Colaboradora en programas radiofónicos y televisivos relacionados con la salud.

- Dr. Alfonso Soler Gomis. Bioquímico. Doctor en Sociología. Profesor de la Facultad de educación y de la facultad de ciencias de la Salud de la Universidad de Alicante. Vicepresidente de la Asociación Gerontológica del Mediterráneo.

\section{SEGUNDA SESIÓN:}

18:20 a 19:20 Mesa de profesionales, docentes y estudiantes de comunicación. Debate

Relación Universidad Empresa y Relaciones Empresa medios de comunicación como vector formación multidisciplinar - transversal y socialmente responsable.

\section{PONENTES:}

- Dra. Estela Bernard. Departamento de comunicación Universidad Jaume I. Ética en la comunicación: Aproximación a la publicidad encubierta.

- Mar Muñoz Rosario. PR-Salud Noticias. La comunicación sobre salud en una agencia como PRNoticias. 
- David Álvarez. Periodista y profesor de la Facultad de Ciencias de la información. La salud y el desarrollo en las organizaciones no gubernamentales.

- Dra. Rosa María Torres. Departamento de comunicación y psicología social. Universidad de Alicante. La comunicación de proximidad y socialmente responsable en el ámbito de la salud nutricional.

- Greengorcer: Un nuevo enfoque comunicativo socialmente responsable en el ámbito de la alimentación saludable.

- Alumnos UCM participantes en organización de evento. Los aprendizajes.

19:20 Clausura y lectura de conclusiones.

Tras la realización del evento, se pasó un cuestionario con preguntas abiertas a los alumnos y alumnas participantes. Las preguntas fueron:

\section{Antes de la experiencia:}

- ¿Qué idea/noción tenías sobre "comunicación para la salud” y sobre comunicación publicitaria de productos alimenticios?

\section{Después de la experiencia:}

- ¿Qué conclusión extraes sobre "comunicación para la salud" y sobre comunicación publicitaria de productos alimenticios?

- ¿Aprecias tras la participación en parte de la campaña de los Powerpupis, un “diálogo de saberes"? ¿Te produce alguna curiosidad investigadora?

3.2. Investigación sobre la experiencia de aprendizaje: los principales resultados

En el apartado de metodología se expuso el resultado de la dinámica de grupos on line antes de la ejecución de la jornada. Por tanto exponemos resultados en relación con el cuestionario pasado tras la experiencia.

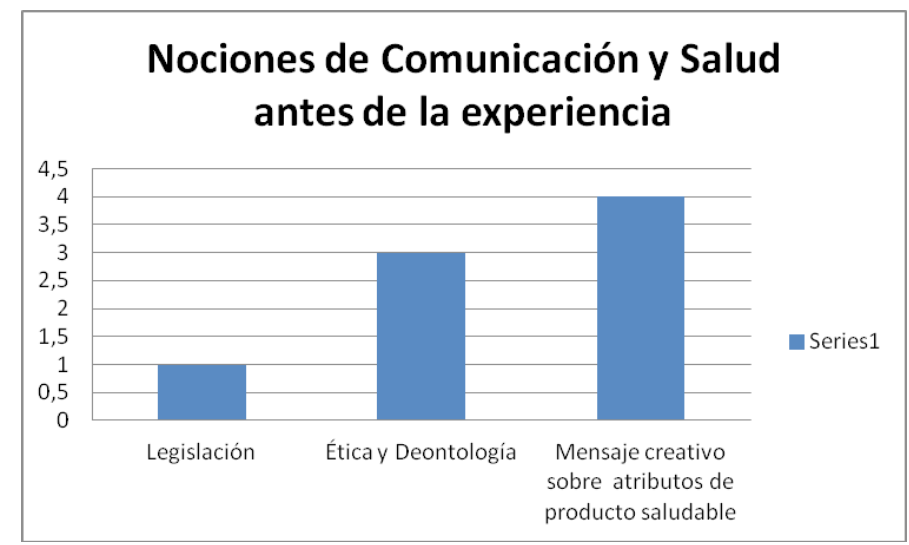

Gráfica 1. Fuente elaboración propia. 
De los 8 estudiantes encuestados 1 relacionaba el concepto de comunicación para la salud y comunicación publicitaria de productos alimenticios con legislación, 3 con ética y deontología y 4 con la elaboración de mensajes creativos sobre atributos de productos alimenticios saludables.

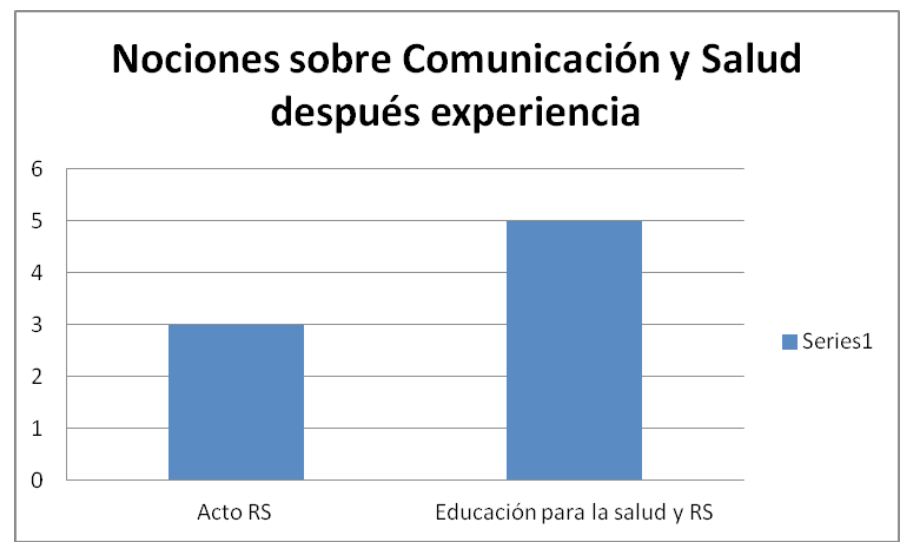

Gráfica 2. Fuente elaboración propia.

De los 8 estudiantes encuestados, 3 definen, tras la experiencia, el concepto de comunicación para la salud y comunicación publicitaria de productos alimenticios como un acto de Responsabilidad Social y 5 como educación para la salud y Responsabilidad Social.

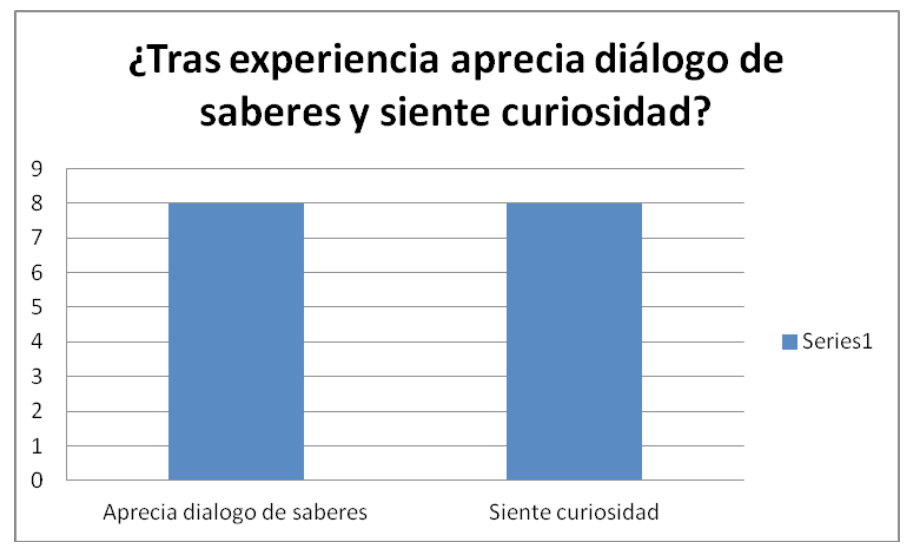

Gráfica 3. Fuente elaboración propia

Los 8 estudiantes encuestados, tras la experiencia, afirman percibir diálogo de saberes en la actividad y sentir curiosidad por ampliar información y hacer más investigación formativa. 
En relación con la empresa cliente, el director general afirma que ve la necesidad de definir claramente el concepto saludable y sus implicaciones en el mensaje publicitario de productos alimenticios. El resto de equipo de la empresa califica de estrategia interesante para la captación de nuevos clientes la celebración de jornadas de tipo científico. Sorprendió gratamente la entrega de alumnas y alumnos participantes, manifestando nuevamente el director la posibilidad de oportunidad laboral en la empresa de algunos de los estudiantes participantes.

\section{Conclusiones}

La unión de diferentes disciplinas, del ámbito publicitario, relaciones públicas, comunicación social, hace que los alumnos aprendan y se motiven más que con un solo enfoque. En este sentido parece pertinente recordar que la educación como proceso formal institucionalizado también debe orientarse a "la creación de aprendizaje de los procesos de generación de conocimiento" (Martínez, 2008) y la creación de comunidades de práctica, "es un reto necesario a asumir por todos los profesionales de la educación y la formación, dado que a través de ellas se puede, por una parte, transferir y generar nuevo conocimiento y por otra, lograr mejores prácticas en el campo de la investigación y la docencia" (Bozu y Imbernón, 2009).

En relación con los objetivos de investigación planteados, se exponen las siguientes conclusiones:

- Los debates propiciaron la aplicación práctica de teorías y conceptos aplicados en el aula.

- Las dinámicas on line y presenciales facilitaron debatir sobre el significado de cuarta cultura y diálogo de saberes como estrategia basada en la gestión de conocimientos integrados a través de la visión multidisciplinar.

- El realizar un caso real y trabajar con clientes reales, entablando conversación con ellos y resolviendo sus problemas permitió formar a los alumnos en los contenidos de publicidad yrelaciones públicas y dotó de seguridad a los alumnos.

- La empresa Cliente se mostró satisfecha con el trabajo de alumnas y alumnos, y comprueba la importancia del marco Universitario y la comunicación de su producto basada en la cultura científica a fin de evitar el "engaño redaccional".

- A raíz de la experiencia, los alumnos y alumnas participantes amplían las nociones sobre comunicación de y para la salud en el ámbito de la publicidad de productos alimentarios.

La hipótesis inicial se confirma: Se estimula su capacidad investigadora multidisciplinar y su capacidad de visión crítica si forman parte de un proceso de investigación acción en el doble papel de observadores y observados. 
El trabajo nos ha permitido constatar, que una actividad práctica, multidisciplinar y de entrenamiento en la organización de una jornada con científicos para comunicar sobre un producto, ha servido de plataforma de lanzamiento de perfiles profesionales del ámbito de la comunicación, posibilitando con ello la visibilidad de los estudiantes ante la empresa cliente, futura empleadora potencial, lo que a su vez ha sido muy motivador para los docentes y alumnos participantes.

Los resultados alcanzados, sugieren que convendría continuar el estudio, ampliando número de participantes, dotando de mayor complejidad el trabajo en base una campaña integral, como forma de estimular en las aulas tanto a docentes como estudiantes la visión crítica y multidisciplinar para una comunicación responsable.

\section{Bibliografía}

BALAS LARA, M. (2011). La gestión de comunicación en el tercer sector. Madrid: ESIC.

BARRANCO SAIZ, F. J. (2005). Marketing social corporativo. La acción social de la empresa. Madrid: ESIC.

BENET, V. J.; NOS ALDÁS, E. (2003). La publicidad en el tercer sector. Tendencias y perspectivas de la comunicación solidaria. Castellón: Icaria.

BOZU, Z.; IMBERNÓN MUÑOZ, F. (2009). "Creando comunidades de práctica y conocimiento en la universidad: Una experiencia de trabajo entre las universidades de lengua catalana". En Revista De Universidad y Sociedad Del Conocimiento, RUSC, vol. 6, $\mathrm{n}^{\circ}$ 1. [Documento en línea recuperado el 11/08/2013 de www.redalyc.org/articulo.oa? $\mathrm{id}=78011179004 \& \mathrm{idp}=1 \& \mathrm{cid}=4407472]$.

CASTILLO, A. (2010). Introducción a las relaciones públicas. España: Instituto de Investigación en Relaciones Públicas.

DÍAZ DE SALAS, S. A.; MENDOZA MARTÍNEZ, V. M.; PORRAS MORALES, C. M. (2011). "Una guía para la elaboración de estudios de caso". En Revista Razón y Palabra, $\mathrm{n}^{\circ}$ 75, febrero - abril 2011. [Documento en línea recuperado el 02/08/2013 de www.razonypalabra.org.mx/N/N75/varia_75/01_Diaz_V75.pdf].

GONZÁLEZ Y TISCAR LARA, A. (2011). "Aprender en la incertidumbre nuevos valores y métodos para formar a los profesionales". En Organización de los estados iberoamericanos. [Documento en línea recuperado el 10/08/2013 de http:// redesoei.ning.com/profiles/blogs/aprender-en-la-incertidumbre-nuevos-valores-y-m-todos-para-formar].

JUHOLIN, E. (2006). Communicare. Viestintä strategiasta käytäntöön. Porvoo (Finlandia): Infor.

KAZEZ R. (2009). "Los estudios de casos y el problema de la selección de la muestra. Aportes del sistema de matrices de datos". En Revista Subjetividad y Procesos Cognitivos, $\mathrm{n}^{\mathrm{o}}$ 13, 71-89. [Documento en línea recuperado el 09/08/2013 de dialnet.unirioja.es/servlet/articulo?codigo $=3130865]$.

KOTLER, P.; HESSEKIEL, D.; LEE, N.R. (2012). Lo bueno funciona. Madrid: 
LEHRER, J. (2010). Proust y la neurociencia. Una visión única de ocho artistas fundamentales de la modernidad. Madrid: Editorial Paidós. Lid Editorial Empresarial.

MARTÍNEZ GARCÍA, B. (2008). "El aprendizaje de la cultura y la cultura de aprender en Convergencia". En: Revista De Ciencias Sociales, n 48, p. 287-307.

MARTÍNEZ GARCÍA, B. (2008). "El aprendizaje de la cultura y la cultura de aprender. Convergencia". En Revista de Ciencias Sociales, n 48, 287-307. [Documento en línea recuperado el 20/08/2013 de www.scielo.org.co/pdf/noma/n28/n28a19. pdf].

MULLARAT, R. (2007). En buena compañía. La responsabilidad social de las empresas. Barcelona: Random House Mondadori.

OLMEDOESTRADA, J. C.(2011). “Educación y divulgación de la ciencia: Tendiendo puentes hacia la alfabetización científica". En Revista Eureka Sobre Enseñanza y Divulgación De Las Ciencias, vol. 8, $\mathrm{n}^{\circ}$ 2, 137-148. [Documento en línea recuperado el 08/08/2013 de www.redalyc.org/articulo.oa?id=92017189001\&idp=1\&$\mathrm{cid}=683683]$.

ROBLES CASTRILLO, R. (2005). Diálogos entre saberes cientificos y artísticos. Impresora Feriva S.A. Columbia.

VALLAEYS, F. (2009). "Responsabilidad Social Universitaria: una nueva filosofía de gestión ética e inteligentes para las universidades". En Educación Superior y Sociedad, vol. 13, no 2, p. 191-220.

WILCOX, D. L.; CAMERON G. T.; XIFRA, J. (2006). Relaciones Públicas. Estrategias y Tácticas. Madrid: Pearson Educación.

XIFRA, J. (2007). Técnicas de las relaciones públicas. Barcelona: Editorial UOC.

\section{Las autoras}

Rosa Torres es profesora del departamento de comunicación y psicología social de la Universidad de Alicante y enseña comunicación en títulos de grado y magister en la Universidad de Alicante. Es doctora en Publicidad y Relaciones Públicas, Máster en biomedicina y biotecnología y tiene postgrado en Responsabilidad Social por RedUnirse (PNUD). Sus líneas de investigación publicaciones relacionan la comunicación, con la calidad docente, la Responsabilidad Social, Desarrollo Local, la salud y el fundraising.

Patricia Núñez es profesora de publicidad en la Facultad de Ciencias de la información de la Universidad Complutense. Doctora en publicidad y máster en filosofía. Miembto de diferentes grupos de investigación nacionales e internacionales y directora del grupo de investigación relacionado con infancia y comunicación Branding Kids. Sus publicaciones tieen como líneas de investigación principales, la infancia, nuevas tecnologías, marcas y tercer sector, así como todo lo relacionado con metodologías educativas innovadoras. 
Liisa Hanninen enseña comunicación en títulos de grado y magister en la Facultad de Ciencias de la Información de la UCM. Doctora en Publicidad y Relaciones Públicas y magister en Comunicación Corporativa por la misma universidad, comenzó sus estudios de Periodismo y Ciencias Políticas en la Universidad de Helsinki. Sus publicaciones y líneas de estudio más recientes se centran en la metodología docente responsable, comunicación de entidades no lucrativas y la comunicación medioambiental. 